\title{
Outcome of Patients With Inherited Neurotransmitter Disorders
}

\author{
Dawn Cordeiro, Garrett Bullivant, Ronald D. Cohn, Julian Raiman, \\ Saadet Mercimek-Andrews
}

\begin{abstract}
We report the outcome of 12 patients with inherited neurotransmitter disorders of monoamine, tetrahydrobiopterin and $\gamma$ amino butyric acid metabolisms from a single Inherited Neurotransmitter Disorder Clinic including tyrosine hydroxylase $(n=2)$, aromatic L-amino acid decarboxylase $(n=1)$, 6-pyruvoyltetrahydropterin synthase, dihydropteridine reductase and succinic semialdehyde dehydrogenase deficiencies. Six patients (with 6-pyruvoyltetrahydropterin synthase, dihydropteridine reductase and tyrosine hydroxylase deficiencies) had normal neurodevelopmental outcome on treatment. Tetrahydrobiopterin loading test in newborns with positive newborn screening for phenylketonuria will identify patients with 6-pyruvoyltetrahydropterin synthase and dihydropteridine reductase deficiencies resulting in abnormal neurotransmitter synthesis in the central nervous system in the neonatal period to initiate disease-specific treatment to improve neurodevelopmental outcome.
\end{abstract}

RÉSUMÉ: Évolution de l'état de santé de patients atteints de troubles héréditaires des neurotransmetteurs. Nous voulons faire état de l'évolution de l'état de santé de douze patients atteints de troubles héréditaires affectant le métabolisme des neurotransmetteurs suivants: les monoamines, la tétrahydrobioptérine et l'acide $\gamma$-aminobutyrique, et ce, à partir d'un simple trouble héréditaire affectant les neurotransmetteurs suivants: la tyrosine hydroxylase $(n=2)$; l'acide L-aminé aromatique decarboxylase $(n=1)$; la 6-pyruvoyltétrahydroptérine synthase; la dihydroptéridine reductase; et finalement, la succinate semialdéhyde déshydrogénase. Six patients (déficits de la 6-pyruvoyltétrahydroptérine synthase, de la dihydroptéridine reductase et de la tyrosine hydroxylase) ont montré une évolution neuro-développementale normale à la suite d'un traitement. Un test de charge de la tétrahydrobioptérine chez des nouveaux-nés donnant à voir à la naissance un dépistage positif pour la phénylcétonurie permettra d'identifier des patients atteints de déficits de la 6-pyruvoyltétrahydroptérine synthase et de la dihydroptéridine reductase. De tels déficits entraînent une synthèse anormale des neurotransmetteurs dans le système nerveux central durant la période néonatale. On pourra de la sorte entamer un traitement spécifique afin d'améliorer l'évolution neuro-développementale des patients visés.

Keywords: Inherited neurotransmitter disorders, Levodopa/carbidopa, 5-hydroxytryptophan, Tetrahydrobiopterin, $\gamma$ amino butyric acid doi:10.1017/cjn.2018.266

Can J Neurol Sci 2018; 45: 571-576

Inherited neurotransmitter disorders affecting monoamine, tetrahydrobiopterin and $\gamma$ amino butyric acid (GABA) metabolisms are rare disorders. Catecholamine (dopamine, epinephrine and norepinephrine) and serotonin metabolism disorders include tyrosine hydroxylase (TH) (OMIM\# 605407) (EC 1.14.16.2) and aromatic L-amino acid decarboxylase (AADC) (OMIM\# 608643) (EC 4.1.1.28) and biopterin metabolism disorders include guanosine triphosphate cyclohydrolase I (OMIM\# 128230) (EC 3.5.4.16), 6-pyruvoyltetrahydropterin synthase (PTPS) (OMIM\# 261640) (EC 4.2.3.12), sepiapterin reductase (OMIM\# 612716) (EC 1.1.1.153) and dihydropteridine reductase (DHPR) (OMIM\# 261630) (EC 1.5.1.34) deficiencies. ${ }^{1-3}$ Positive newborn screening for phenylketonuria and tetrahydrobiopterin loading test leading to normalization of plasma phenylalanine levels within 24 hours are diagnostic for PTPS, DHPR and pterin-4acarbinolamine dehydratase deficiencies. ${ }^{1-3}$ Clinical features are usually infantile onset and include tremor, hypokinesia, rigidity, dystonia, chorea, motor and cognitive dysfunction, failure-tothrive, sleep disturbances and autonomic dysfunction (e.g., blood pressure fluctuations, abdominal distention, temperature instability, constipation and diarrhea episodes and fatigue) secondary to catecholamine and serotonin deficiency. ${ }^{1-4}$ Measurement of CSF neurotransmitter metabolites, including homovanillic acid (HVA), 5-hydroxyindolacetic acid (5-HIAA), 3-O-methyldopa (3-OMD), tetrahydrobiopterin, biopterin and neopterin, is a useful diagnostic tool to identify disorders of catecholamine, serotonin and biopterin metabolisms. ${ }^{1,4}$ The diagnosis is confirmed by direct sequencing of candidate genes based on the CSF neurotransmitter metabolite profiles suggestive of a specific enzyme deficiency. ${ }^{1-4}$

$\gamma$ Amino butyric acid metabolism defects are succinic semialdehyde dehydrogenase (SSADH) (OMIM\# 271980) and GABA

From the Department of Pediatrics, Division of Clinical and Metabolic Genetics,

University of Toronto, Toronto, Ontario, Canada (DC, GB, RDC, SM-A); Genetics and Genome Biology Program, Research Institute, The Hospital for Sick Children, Toronto, Ontario, Canada (RDC, SM-A); Department of Pediatrics, University of Toronto, Toronto, Ontario, Canada (RDC, SM-A); Birmingham's Children Hospital, Birmingham, England (JR); Institute of Medical Sciences, University of Toronto, Toronto, Ontario, Canada (SM-A)

Received January 9, 2018. Final Revisions Submitted May 19, 2018. Date of ACCEPTANCE MAY 31, 2018.

Correspondence to: S. Mercimek-Andrews, Division of Clinical and Metabolic Genetics, The Hospital for Sick Children, 555 University Avenue, Toronto, ON, Canada M5G 1X8. Email: saadet.andrews@ sickkids.ca 
transaminase (OMIM\# 613163) (EC 2.6.1.19) deficiencies. ${ }^{2}$ Neonatal-onset seizures are clinical features of GABA transaminase deficiency. Infantile-onset hypotonia, global developmental delay and/or seizures are clinical features of SSADH deficiency. Diagnosis of SSADH deficiency is suspected by elevated 4-hydroxybutyrate in urine organic acid analysis, whereas GABA transaminase deficiency is suspected by elevated GABA and $\beta$-alanine levels in CSF amino acid analysis. ${ }^{2}$ The diagnosis is confirmed by direct sequencing of candidate genes.

In this study, we report outcome of patients with pediatric inherited neurotransmitter disorders affecting monoamine, tetrahydrobiopterin and GABA metabolisms from a single Inherited Neurotransmitter Disorder Clinic to increase our knowledge regarding those rare disorders.

This study was approved by the Institutional Research Ethics Board (Approval\# 1000057003). All patients with disorders of monoamine, tetrahydrobiopterin and GABA metabolisms seen in our Inherited Neurotransmitter Disorder Clinic were included. All patients were referred for the management and treatment after their suspected diagnosis. We reviewed electronic patient charts for clinical features, neuroimaging, biochemical investigations, molecular genetic investigations and treatment. Targeted direct sequencing of relevant genes was performed in various clinical molecular genetics laboratories according to their methods.

In total, 12 patients with inherited neurotransmitter disorders affecting monoamine, tetrahydrobiopterin and GABA metabolisms diagnosed during January 1999 and March 2017 were included. Demographics, clinical features, biochemical and molecular genetic data for all patients are listed in Table 1a. Three patients had primary disorders of monoamine metabolism including TH deficiency $(n=2)$ (one patient reported previously ${ }^{5}$ ) and AADC deficiency $(n=1)$. Seven patients had disorders of tetrahydrobiopterin metabolism including PTPS deficiency $(n=4)$ (one patient reported previously ${ }^{5}$ ) and DHPR deficiency $(n=3)$ (one patient reported previously ${ }^{5}$ ). Six out of these seven patients were identified by positive newborn screening for phenylketonuria. Owing to normalization of phenylalanine levels after tetrahydrobiopterin loading test, further investigations including elevated urine neopterin (biomarker for PTPS deficiency) or deficient DHPR activity in blood dot spot confirmed the biochemical diagnosis in the neonatal period in six patients. Their genetic diagnosis was confirmed by direct sequencing of $P T S$ (for PTPS deficiency) and QDPR (for DHPR deficiency), respectively. One patient with DHPR deficiency was initially treated as if the disorder was phenylketonuria. The correct diagnosis of DHPR deficiency was made at 11 months of age when the child had global developmental delay and seizures. Two patients were diagnosed by low CSF HVA suggestive of TH deficiency. One patient was diagnosed by low HVA and 5-HIAA and high 3-OMD suggestive of AADC deficiency. Genetic diagnosis was confirmed by direct sequencing of $T H$ in two patients. One patient with AADC deficiency underwent whole-exome sequencing in addition to CSF neurotransmitter analysis requested at the same time, confirming the genetic diagnosis.

Two patients had SSADH deficiency, which was identified by the presence of elevated 4-hydroxybutyric acid in urine organic acid analysis. Direct sequencing of ALDH5A1 (a known pathogenic variant ${ }^{6}$ ) confirmed the diagnosis of SSADH deficiency in both patients. In patient 11, quantification of 4-hydroxybutyric acid in urine was markedly elevated $(119 \mathrm{mmol} / \mathrm{mol}$ creatinine; reference range 0.09-5). His SSADH activity was non-detectable (reference range 1907-3901 pmol/minute/mg protein) in the cultured lymphoblasts. In additional repeat urine organic acid screens, one demonstrated elevated 4-hydroxybutyric acid and the other was normal.

Treatment and treatment outcomes are listed in Table $1 \mathrm{~b}$. Neuropsychological assessment results of patients older than 3 years of age are summarized in Table 1c. Three patients with PTPS, two patients with DHPR and one patient with TH deficiencies had age-appropriate developmental outcome. Because of late diagnosis and/or compliance problems with L-dopa/carbidopa and 5-hydroxytryptophan, one patient with DHPR and one patient with PTPS deficiencies did not achieve normal neurodevelopmental outcome (Table 1c). In one patient with $\mathrm{TH}$ and in one patient with AADC deficiencies, medical management resulted in improvements of movement disorders but did not improve neurodevelopmental outcome. All patients with PTPS, DHPR and TH deficiencies were monitored by prolactin levels and maintained normal prolactin levels during outpatient follow-up clinic visits. All patients with PTPS deficiency were on tetrahydrobiopterin once or twice a day and monitored by plasma and blood dot spot phenylalanine target level $<360 \mu \mathrm{mol} / \mathrm{L}$. All patients with DHPR deficiency were on the phenylalaninerestricted diet and achieved plasma phenylalanine target level $<360 \mu \mathrm{mol} / \mathrm{L}$ throughout the therapy.

The PTPS deficiency was the most common inherited neurotransmitter disorder in our study. The homozygous p.Gly315Ser variant in $T H$ in two unrelated families and the homozygous p.Thr67Met variant in PTS in two cousins from non-consanguineous parents were reported in patients from Sri Lankan ethnic background. These variants are likely to be common founder variants in this population. We report normal neurodevelopmental and neurocognitive outcome in a patient with DHPR deficiency with excellent treatment compliance for 18 years. So far, less than ten patients with DHPR deficiency have been reported for their detailed treatment outcome. Our study reports two additional DHPR patients' longterm treatment outcome and normal neurocognitive functions in one of those patients. Good treatment compliance is the key for normal neurodevelopmental outcome.

Sixty-eight patients (sum of patients with DHPR and PTPS deficiencies) (12\%) were treated from the neonatal period reported in The International Database of Tetrahydrobiopterin Deficiencies. $^{3}$ Developmental delay was present in $28 \%$, seizures in $5 \%$, dystonia in $5 \%$, other movement disorders in $20 \%$ and autonomic dysfunction in $10 \%$ of those patients. In 624 patients, treatment was initiated after the neonatal period. In the latter group, developmental delay was present in $48 \%$, seizures in $28 \%$ and autonomic dysfunction in $15 \%$ of those patients. The percentage of patients with dystonia and other movement disorders were similar to the patients in whom treatment was initiated in the neonatal period. ${ }^{3}$ More than $50 \%$ of the patients, treated from the neonatal period, had no symptoms. However, $75 \%$ of the patients, treated after the neonatal period, were symptomatic. ${ }^{3}$ In our study, $86 \%(6 / 7)$ of the patients were treated from the newborn period and $83 \%(5 / 6)$ of these patients had normal neurodevelopmental outcome. Tetrahydrobiopterin loading test or biochemical investigations including urine neopterin and DHPR activity in blood dot spot in newborns with positive newborn screening for phenylketonuria are essential to confirm diagnosis and initiate treatment in the neonatal period for PTPS and DHPR deficiencies. 
Table 1a: Clinical features, neuroimaging, biochemical and molecular genetics results of patients with inherited metabolic disorders presenting with inherited neurotransmitter disorders

\begin{tabular}{|c|c|c|c|c|c|c|}
\hline $\begin{array}{l}\text { Patient \#/sex/ } \\
\text { current age } \\
\text { reference }\end{array}$ & $\begin{array}{l}\text { Diagnosis/age of onset/age of } \\
\text { diagnosis }\end{array}$ & $\begin{array}{l}\text { Clinical features at } \\
\text { presentation }\end{array}$ & Brain MRI/MRS & $\begin{array}{l}\text { Diagnostic biochemical } \\
\text { investigations (results) }\end{array}$ & $\begin{array}{c}\text { CSF HVA/5-HIAA/3- } \\
\text { OMD/THB/NeP at } \\
\text { diagnosis }\end{array}$ & Molecular genetic test results reference \\
\hline $1 / \mathrm{F} / 1 \mathrm{y}$ & $\begin{array}{l}\text { PTPS deficiency/newborn } \\
\text { screening/7 days }\end{array}$ & Dystonia, FTT & NP & $\begin{array}{l}\uparrow \text { Phe }(1636) \text {, } \text { urine neopterin/ } \\
\text { biopterin ratio }(>200)\end{array}$ & $\downarrow 143 / \downarrow 84 / \mathrm{N} / \downarrow 15 / \uparrow 171$ & $\begin{array}{l}\text { Novel compound heterozygous } \\
\text { c.151C }>\mathrm{T} \text { (p.His51Tyr)/c.34G > A (p.Ala12Thr) } \\
\text { in PTS (parents heterozygous) }\end{array}$ \\
\hline $2 / \mathrm{F} / 2$ y $10 \mathrm{~m}^{*}$ & $\begin{array}{l}\text { PTPS deficiency/newborn } \\
\text { screening/9 days }\end{array}$ & Dystonia, IUGR, FTT & NP & 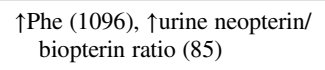 & $\begin{array}{l}\text { N } 406 / \downarrow 194 / \mathrm{N} / \downarrow 14 / \\
\quad \uparrow 260\end{array}$ & $\begin{array}{l}\text { Known homozygous c.200C }>\mathrm{T} \text { (p.Thr67Met) in } \\
P T S^{9} \text { (parents heterozygous) }\end{array}$ \\
\hline $3 / \mathrm{F} / 10 \mathrm{~m}^{*}$ & $\begin{array}{l}\text { PTPS deficiency/newborn } \\
\text { screening/8 days }\end{array}$ & Dystonia, IUGR & NP & $\begin{array}{l}\uparrow \text { Phe (2094), } \text { 个urine neopterin/ } \\
\text { biopterin ratio (50) }\end{array}$ & $\downarrow 256 / \downarrow 99 / \mathrm{N} / \downarrow 17 / \uparrow 201$ & $\begin{array}{l}\text { Known homozygous c.200C }>\mathrm{T} \text { (p.Thr67Met) in } \\
\text { PTS } 9 \text { (parents heterozygous) }\end{array}$ \\
\hline $4 / F / 6 y^{9}$ & $\begin{array}{l}\text { PTPS deficiency/newborn } \\
\text { screening/12 days }\end{array}$ & $\begin{array}{l}\text { Dystonia, IUGR, } \\
\text { preterm }\end{array}$ & Mild cerebral atrophy/NP & $\begin{array}{l}\uparrow \text { Phe (1780), †urine neopterin/ } \\
\text { biopterin ratio (109) }\end{array}$ & $\downarrow 157 / \downarrow 78 / \mathrm{N} / \mathrm{N} 69 / \uparrow 199$ & $\begin{array}{l}\text { Known homozygous c. } 200 \mathrm{C}>\mathrm{T} \text { (p.Thr67Met) in PTS } \\
\text { (parents heterozygous) }\end{array}$ \\
\hline $5 / F / 18$ y & $\begin{array}{l}\text { DHPR deficiency/newborn } \\
\text { screening/14 days }\end{array}$ & Dystonia & NP & $\begin{array}{l}\uparrow \text { Phe (2618), blood dot spot } \\
\text { DHPR non-detectable }\end{array}$ & NP & $\begin{array}{l}\text { Novel compound heterozygous c.115A }>\mathrm{G} \\
\text { (p.Ser39Gly)/c.661C }>\mathrm{T} \text { (p.Arg221X) in } Q D P R \\
\text { (parents heterozygous) }\end{array}$ \\
\hline $6 / F / 13.5$ y & DHPR deficiency/NA/11 m & GDD, dystonia, seizures & $\begin{array}{l}\text { Right parieto-occipital } \\
\text { encephalomalacia }\end{array}$ & $\mathrm{NA}$ & NP & NA \\
\hline $7 / \mathrm{M} / 1.5$ y & $\begin{array}{l}\text { DHPR deficiency/newborn } \\
\text { screening/8 days }\end{array}$ & Dystonia & NP & $\begin{array}{l}\uparrow \text { Phe (531), blood dot spot } \\
\text { DHPR non-detectable }\end{array}$ & $\downarrow 174 / \downarrow 37 / N / \downarrow 33 / N 13$ & $\begin{array}{l}\text { Novel homozygous c. } 44 \mathrm{~T}>\mathrm{C} \text { (p.Val15A1a) in } Q D P R \\
\text { (parents heterozygous) }\end{array}$ \\
\hline $8 / F / 3.5 y^{* *}$ & TH deficiency $/ 6 \mathrm{~m} / 7 \mathrm{~m}$ & $\begin{array}{l}\text { GDD, dystonia, oral } \\
\text { dyskinesia, truncal } \\
\text { hypotonia }\end{array}$ & Cerebral atrophy/N & $\downarrow$ CSF HVA 19 & $\downarrow 19 / \mathrm{N} 331 / \mathrm{N} / \mathrm{NP} / \mathrm{NP}$ & $\begin{array}{l}\text { Known homozygous c. } 943 \mathrm{G}>\mathrm{A} \text { (p.Gly315Ser) in } \\
T H^{9} \text { (parents heterozygous) }\end{array}$ \\
\hline $9 / \mathrm{F} / 6$ y9** & TH deficiency $/ 2 \mathrm{~m} / 2$ y $2 \mathrm{~m}$ & $\begin{array}{l}\text { GDD, dystonia, chorea, } \\
\text { microcephaly, } \\
\text { hypotonia }\end{array}$ & $\begin{array}{l}\text { Bilateral increased } \mathrm{T} 2 \\
\text { signal in the } \mathrm{GB} / \mathrm{mild} \\
\text { NAA decrease }\end{array}$ & $\downarrow$ CSF HVA 7 & $\downarrow 7 / \mathrm{N} 370 / \mathrm{N} / \mathrm{NP} / \mathrm{NP}$ & $\begin{array}{l}\text { Known homozygous c. } 943 \mathrm{G}>\mathrm{A} \text { (p.Gly315Ser) in } T H \\
\text { (parents heterozygous) }\end{array}$ \\
\hline $10 / F / 3$ y & AADC deficiency $/ 2 \mathrm{~m} / 11 \mathrm{~m}$ & $\begin{array}{l}\text { GDD, dystonia, } \\
\text { oculogyric crises, } \\
\text { hypotonia, irritability }\end{array}$ & $\mathrm{N} / \mathrm{N}$ & $\downarrow$ RBC AADC 1.8 & $\downarrow 25 / \downarrow 13 / \uparrow 1609 / \mathrm{NP} / \mathrm{NP}$ & $\begin{array}{l}\text { Novel compound heterozygous c.665T }>\mathrm{C} \\
\text { (p.Leu222Pro)/ c.286G }>\text { A (p.Gly96Arg) in } D D C \\
\text { (parents heterozygous) }\end{array}$ \\
\hline $11 / \mathrm{M} / 17 \mathrm{y}$ & SSADH deficiency/first year/6 y & GDD, ataxia, psychosis & $\begin{array}{l}\text { Symmetrical increased } \\
\text { signal intensity in GB } \\
\text { and DN/elevated GLT } \\
\text { and GLU }\end{array}$ & †urine 4-HBA & NP & $\begin{array}{l}\text { Known homozygous c. } 278 \mathrm{G}>\mathrm{T} \text { (p.Cys93Phe) in } \\
A L D H 5 A 1^{8} \text { (parents heterozygous) }\end{array}$ \\
\hline $12 / \mathrm{F} / 6 \mathrm{y}^{* *}$ & SSADH deficiency $/ 6 \mathrm{~m} / 11 \mathrm{~m}$ & GDD, hypotonia & Cerebral atrophy/NP & $\begin{array}{l}\text { †urine 4-HBA, 4-HBA lactone, } \\
\text { 3,4-(OH) })_{2} \text {-BA }\end{array}$ & NP & $\begin{array}{l}\text { Known homozygous c. } 1226 \mathrm{G}>\mathrm{A} \text { (p.Gly409Asp) in } \\
A L D H 5 A I^{8} \text { (parents heterozygous) }\end{array}$ \\
\hline
\end{tabular}

$\uparrow=$ elevated; $\downarrow=$ decreased; AADC $=$ aromatic $\mathrm{L}$-amino acid decarboxylase; $\mathrm{d}=$ day(s); DHPR $=$ dihydropteridine reductase; FTT = failure to thrive; GB $=$ globus pallidus; GDD $=$ global developmental delay; GLT $=$ glutamate; $\mathrm{GLU}=$ glutamine; 4-HBA = 4-hydroxybutyric acid; 5-HIAA = 5-hydroxyindol acetic acid; HVA = homovanillic acid; $\mathrm{m}=$ month(s); IUGR = intrauterine growth retardation; MRS = magnetic resonance spectroscopy; $\mathrm{N}=$ normal; NA = not available; NeP=neopterine; NP=not performed; 3-OMD=3-O-methyldopa; Phe=phenylalanine; PTPS =6-pyruvoyltetrahydropterin synthase; RBC=red blood cell; SSADH = succinic semialdehyde dehydrogenase; $\mathrm{THB}=$ tetrahydrobiopterine; $\mathrm{TH}=$ tyrosine hydroxylase; $\mathrm{y}=$ year(s).

*Fathers are brothers.

**Parents are first cousins.

Age-appropriate reference ranges for biochemical investigations: Plasma phenylalanine $45-65 \mu \mathrm{mol} / \mathrm{L}$; urine neopterin/biopterin ratio 0.2-3.0; blood dot spot DHPR activity 7-22 $\mu \mathrm{mol} / \mathrm{g}$ hb; AADC RBC activity 36-129 pmol/minute/ml. CSF neurotransmitters age-appropriate reference range: CSF HVA: 0-0.2 years $=337-1299 \mathrm{nmol} / \mathrm{L} ; 0.2-0.5$ years $=450-1132 \mathrm{nmol} / \mathrm{L} ; 0.5-2$ years $=294-1115 \mathrm{nmol} / \mathrm{L} ; 2-5$ years $=233-928 \mathrm{nmol} / \mathrm{L} ; 5-10$ years $=218-852 \mathrm{nmol} / \mathrm{L} ; 10-15$ years $=167-563 \mathrm{nmol} / \mathrm{L} ;$ adults $=145-324 \mathrm{nmol} / \mathrm{L}$

CSF 5HIAA: 0-0.2 years $=208-1159 \mathrm{nmol} / \mathrm{L} ; 0.2-0.5$ years $=179-711 \mathrm{nmol} / \mathrm{L} ; 0.5-2$ years $=129-520 \mathrm{nmol} / \mathrm{L} ; 2-5$ years $=74-345 \mathrm{nmol} / \mathrm{L} ; 5-10$ years $=66-338 \mathrm{nmol} / \mathrm{L} ; 10-15 \mathrm{years}=67-189 ;$ adults $=67-140 \mathrm{nmol} / \mathrm{L}$.

CSF 3-O-MD: $0-0.2$ years $\leq 300 \mathrm{nmol} / \mathrm{L} ; 0.2-0.5$ years $\leq 300 \mathrm{nmol} / \mathrm{L} ; 0.5-2$ years $\leq 300 \mathrm{nmol} / \mathrm{L} ; 2-5$ years $\leq 150 \mathrm{nmol} / \mathrm{L} ; 5-10$ years $\leq 100 \mathrm{nmol} / \mathrm{L} ; 10-15$ years $\leq 100 \mathrm{nmol} / \mathrm{L} ;$ adults $\leq 100 \mathrm{nmol} / \mathrm{L}$.

CSF tetrahydrobiopterin: $0-0.2$ years $=40-105 \mathrm{nmol} / \mathrm{L} ; 0.2-0.5$ years $=23-98 \mathrm{nmol} / \mathrm{L} ; 0.5-2$ years $=18-58 \mathrm{nmol} / \mathrm{L} ; 5-10$ years $=9-40 \mathrm{nmol} / \mathrm{L} ; 10-15$ years $=9-32 \mathrm{nmol} / \mathrm{L} ;$ adults $=10-30 \mathrm{nmol} / \mathrm{L}$

CSF neopterin: $0-0.2$ years $=7-65 \mathrm{nmol} / \mathrm{L} ; 0.2-0.5$ years $=7-65 \mathrm{nmol} / \mathrm{L} ; 0.5-2$ years $=7-65 \mathrm{nmol} / \mathrm{L} ; 2-5$ years $=7-65 \mathrm{nmol} / \mathrm{L} ; 5-10$ years $=7-40 \mathrm{nmol} / \mathrm{L} ; 10-15$ years $=8-33 \mathrm{nmol} / \mathrm{L} ;$ adults $=8-28 \mathrm{nmol} / \mathrm{L}$. 
Table 1b: Treatment and treatment outcome of patients with inherited neurotransmitter disorders are listed

\begin{tabular}{|c|c|c|c|}
\hline Patient \#/diagnosis/current age reference & Treatment (duration of treatment) & Management implications of diagnosis & Outcome \\
\hline 1/PTPS deficiency $/ 5 \mathrm{~m}$ & $\begin{array}{l}\text { L-dopa/carbidopa } 10 \mathrm{mg} / \mathrm{kg} / \mathrm{d}(5 \mathrm{mg} / 1.25 \mathrm{mg} / \mathrm{ml}) \\
5 \text {-HTP } 10 \mathrm{mg} / \mathrm{kg} / \mathrm{d} \\
\text { Tetrahydrobiopterine } 10 \mathrm{mg} / \mathrm{d} \\
\text { Folinic acid } 5 \mathrm{mg} / \mathrm{d}\end{array}$ & Resolved dystonia, resolved FTT, normal Phe levels & Age-appropriate development \\
\hline 2/PTPS deficiency $/ 2$ y $4 \mathrm{~m}$ & $\begin{array}{l}\text { L-dopa/carbidopa } 10 \mathrm{mg} / \mathrm{kg} / \mathrm{d}(5 \mathrm{mg} / 1.25 \mathrm{mg} / \mathrm{ml}) \\
5 \text {-HTP } 10 \mathrm{mg} / \mathrm{kg} / \mathrm{d} \\
\text { Tetrahydrobiopterine } 10 \mathrm{mg} / \mathrm{d} \\
\text { Folinic acid } 5 \mathrm{mg} / \mathrm{d}\end{array}$ & Resolved dystonia, resolved FTT, normal Phe levels & Age-appropriate development \\
\hline 3/PTPS deficiency/3.5 m & $\begin{array}{l}\text { L-dopa/carbidopa } 10 \mathrm{mg} / \mathrm{kg} / \mathrm{d}(5 \mathrm{mg} / 1.25 \mathrm{mg} / \mathrm{ml}) \\
5 \text {-HTP } 10 \mathrm{mg} / \mathrm{kg} / \mathrm{d} \\
\text { Tetrahydrobiopterine } 10 \mathrm{mg} / \mathrm{d} \\
\text { Folinic acid } 5 \mathrm{mg} / \mathrm{d}\end{array}$ & Resolved dystonia, resolved FTT, normal Phe levels & Age-appropriate development \\
\hline 4/PTPS deficiency/5.5 y & $\begin{array}{l}\text { L-dopa/carbidopa } 10 \mathrm{mg} / \mathrm{kg} / \mathrm{d}(5 \mathrm{mg} / 1.25 \mathrm{mg} / \mathrm{ml}) \\
5 \text {-HTP } 10 \mathrm{mg} / \mathrm{kg} / \mathrm{d} \\
\text { Tetrahydrobiopterine } 10 \mathrm{mg} / \mathrm{d} \\
\text { Folinic acid } 5 \mathrm{mg} / \mathrm{d}\end{array}$ & Resolved dystonia, FTT, normal Phe levels & GDD owing to treatment compliance problems \\
\hline 5/DHPR deficiency/17 y $10 \mathrm{~m}$ & $\begin{array}{l}\text { L-dopa/carbidopa } 10 \mathrm{mg} / \mathrm{kg} / \mathrm{d}(5 \mathrm{mg} / 1.25 \mathrm{mg} / \mathrm{ml}) \\
5 \text {-HTP } 10 \mathrm{mg} / \mathrm{kg} / \mathrm{d} \\
\text { Folinic acid } 5 \mathrm{mg} / \mathrm{d} \\
\text { Phe-restricted diet }\end{array}$ & Resolved dystonia, normal Phe levels & Normal cognitive functions (except impairment in mathematics) \\
\hline 6/DHPR deficiency/12 y $11 \mathrm{~m}$ & $\begin{array}{l}\text { L-dopa } / \text { carbidopa } 10 \mathrm{mg} / \mathrm{kg} / \mathrm{d}(5 \mathrm{mg} / 1.25 \mathrm{mg} / \mathrm{ml}) \\
5 \text {-HTP } 10 \mathrm{mg} / \mathrm{kg} / \mathrm{d} \\
\text { Folinic acid } 5 \mathrm{mg} / \mathrm{d} \\
\text { Phe-restricted diet }\end{array}$ & Bilateral hand dystonia, normal Phe levels & Moderate ID owing to treatment compliance problems \\
\hline 7/DHPR deficiency/1 y & $\begin{array}{l}\text { L-dopa/carbidopa } 10 \mathrm{mg} / \mathrm{kg} / \mathrm{d}(5 \mathrm{mg} / 1.25 \mathrm{mg} / \mathrm{ml}) \\
5 \text {-HTP } 10 \mathrm{mg} / \mathrm{kg} / \mathrm{d} \\
\text { Folinic acid } 5 \mathrm{mg} / \mathrm{d} \\
\text { Phe-restricted diet (intake of Phe/day) }\end{array}$ & Resolved dystonia, normal Phe levels & Age-appropriate development \\
\hline $8 / \mathrm{TH}$ deficiency/3 y & $\begin{array}{l}\text { L-dopa/carbidopa } 10 \mathrm{mg} / \mathrm{kg} / \mathrm{d} \\
\text { Folinic acid } 5 \mathrm{mg} / \mathrm{d}\end{array}$ & Intermittent dystonia during infections & Age-appropriate development \\
\hline 9/TH deficiency $/ 5$ y $3 \mathrm{~m}^{8}$ & $\begin{array}{l}\text { L-dopa/carbidopa } 10 \mathrm{mg} / \mathrm{kg} / \mathrm{d} \\
\text { Folinic acid } 5 \mathrm{mg} / \mathrm{d}\end{array}$ & Spasticity & Severe GDD \\
\hline 10/AADC deficiency $/ 2$ y $5 \mathrm{~m}$ & $\begin{array}{l}\text { Trihexyphenydil } 20 \mathrm{mg} / \mathrm{d} \\
\text { Pramipexole } 75 \mu \mathrm{\mu g} / \mathrm{kg} / \mathrm{d} \\
\text { Tranylcipromine } 0.5 \mathrm{mg} / \mathrm{kg} / \mathrm{d} \\
\text { Pyridoxine } 200 \mathrm{mg} / \mathrm{d}\end{array}$ & Mildly improved oculogyric crises & Severe GDD \\
\hline
\end{tabular}

$\mathrm{AADC}=$ aromatic L-amino acid decarboxylase; $\mathrm{d}=$ day(s); DHPR = dihydropteridine reductase; FTT = failure to thrive; GDD = global developmental delay; 5-HTP = 5-hydroxytryptophan; $\mathrm{ID}=$ intellectual disability; $\mathrm{m}=$ month(s); PTPS $=6$-pyruvoyltetrahydropterin synthase $\mathrm{TH}=$ tyrosine hydroxylase; $\mathrm{y}=$ year(s). 
Table 1c: Neuropsychological assessment results of patients with inherited neurotransmitter disorders are summarized

\begin{tabular}{|c|c|c|}
\hline Patient study ID/diagnosis/age of assessment & Neuropsychological assessment & Classification/percentiles (average $25-75$ th percentiles) \\
\hline \multirow[t]{2}{*}{ 4/PTPS deficiency/3 y } & Mullen Scales of Early Learning & $\begin{array}{l}\text { VRS }=1 \text { st (very low) } \\
\text { FMS }=1 \text { st (very low) } \\
\text { RLS }=<1 \text { st (very low) } \\
\text { ELS }=<1 \text { st (very low) } \\
\text { ELC }=<1 \text { st (very low) }\end{array}$ \\
\hline & Vineland Adaptive Behaviour Scales-II ${ }^{4}$ & $\begin{array}{l}\mathrm{C}=5 \text { th (moderately low) } \\
\text { DLS }=8 \text { th (moderately low) } \\
\mathrm{S}=30 \text { th (adequate) } \\
\mathrm{M}=7 \text { th (moderately low) } \\
\mathrm{ABC}=7 \text { th (moderately low) }\end{array}$ \\
\hline \multirow[t]{3}{*}{ 5/DHPR deficiency/17 y $10 \mathrm{~m}$} & $\begin{array}{l}\text { Wechsler Adult Intelligence Scale-IV Verbal Comprehension index } \\
\text { Perceptual Reasoning index } \\
\text { Working memory index } \\
\text { Processing speed index }\end{array}$ & $\begin{array}{l}21 \text { (low average) } \\
\quad<1 \text { (impaired) } \\
<1 \text { (impaired) } \\
4 \text { (borderline impaired) }\end{array}$ \\
\hline & Academic achievement (WIAT-III) & $\begin{array}{l}\text { Word reading }=34 \text { (average) } \\
\text { Pseudoword decoding }=25 \text { (average) } \\
\text { Spelling }=27 \text { (average) } \\
\text { Reading comprehension }=34 \text { (average) } \\
\text { Math problem solving }=<1 \text { (impaired) }\end{array}$ \\
\hline & Language skills (PPVT-4) & 25 (average) \\
\hline \multirow[t]{2}{*}{ 6/DHPR deficiency/9 y $7 \mathrm{~m}$} & $\begin{array}{l}\text { Wechsler Adult Intelligence Scale-IV Full scale IQ } \\
\text { Verbal Comprehension index } \\
\text { Perceptual Reasoning index } \\
\text { Working memory index } \\
\text { Processing speed index }\end{array}$ & $\begin{aligned}< & 1 \text { (extremely low) } \\
& <1 \text { (extremely low) } \\
& <1 \text { (extremely low) } \\
& <1 \text { (extremely low) } \\
& <1 \text { (extremely low) }\end{aligned}$ \\
\hline & Academic achievement (WIAT-III) & $\begin{array}{l}\text { Word reading }=10 \text { (low average) } \\
\text { Spelling }=19 \text { (low average) } \\
\text { Reading comprehension }=3 \text { (borderline impaired) } \\
\text { Math problem solving }=<1 \text { (extremely low) }\end{array}$ \\
\hline
\end{tabular}

$\mathrm{ABC}=$ adaptive behavior composite; $\mathrm{C}=$ communication; $\mathrm{DHPR}=$ dihydropteridine reductase; $\mathrm{DLS}=$ daily living skills; ELS = expressive language score; ELC = early learning composite; FMS = fine motor score; $\mathrm{m}=$ month(s); $\mathrm{M}=$ motor skills; PPVT-4 = Peabody Picture Vocabulary Test-Fourth Edition; PTPS = 6-pyruvoyltetrahydropterin synthase; RLS = receptive language score; $\mathrm{S}=$ socialization; VRS = visual reception score; WIAT-III = Wechsler Individual Achievement Test-Third Edition; y = year(s).

Mullen Scales of Early Learning normal scores = average 25-74th percentiles. ELC is the total score of all visual, fine motor, receptive language and expressive language. All ELC scores are calculated without the gross motor score.

Vineland Adaptive Behaviour Scales-Second Edition normal scores = average 25-74th percentiles.

Wechsler Adult Intelligence Scale-IV normal scores $=$ mean $100 \pm 15$ SD.

Wechsler Individual Achievement Test-Third Edition normal scores $=$ Mean $100 \pm 15$ SD.

Less than 200 patients with SSADH deficiency have been reported. 4-hydroxybutyric acid in urine organic acid analysis and symmetrical increased signal intensity in globus pallidus are typical biochemical and neuroimaging features of SSADH deficiency. ${ }^{2}$ False negative (e.g., hidden 4-hydroxybutyric acid peak by a large urea peak) or false positive (e.g., urine collection by 4 hydroxybutyric acid containing Coloplast SpeediCath catheters) urine organic acid results have been reported to interfere with the diagnosis of SSADH deficiency. ${ }^{2}$ In one of the early case series, several patients were reported to have normal brain MRI. ${ }^{7}$ In our study, one SSADH patient had normal urine organic acid analysis and the second SSADH patient had no basal ganglia changes.

More than 100 patients with AADC deficiency have been reported so far. Consensus guidelines for the diagnosis and treatment of AADC deficiency were published recently. ${ }^{8}$ The treatment consists of pyridoxine, dopamine agonists, monoamine oxidase inhibitors and anticholinergic agents for symptomatic treatment. Unfortunately, this treatment does not improve neurodevelopmental outcome.
Less than 100 patients have been reported with TH deficiency. The phenotype ranges from dopa-responsive dystonia to severe progressive infantile-onset encephalopathy and Parkinsonism. ${ }^{9}$ Patients with severe phenotype develop dopa-induced dyskinesias. ${ }^{9}$ Homozygous or compound heterozygous p.Gly315Ser variant results in variable phenotype and variable response to 1-dopa/carbidopa therapy based on our, and previously reported, cases. ${ }^{10}$

In summary, we report 12 patients with inherited neurotransmitter disorders affecting monoamine, tetrahydrobiopterin and GABA metabolisms and their favorable neurodevelopmental outcome owing to good treatment compliance and early initiation of therapy in patients with PTPS, DHPR and TH deficiencies. All patients with infantile-onset global developmental delay, truncal hypotonia, movement disorders, as well as autonomic dysfunction, should be investigated by CSF neurotransmitter metabolite measurements to be able to initiate early treatment in inherited neurotransmitter disorders affecting monoamine and tetrahydrobiopterin metabolisms. Neither normal urine organic acid 
analysis nor normal neuroimaging would exclude the diagnosis of SSADH deficiency in patients with global developmental delay.

\section{ACKNOWLEDGMENTS}

The authors thank Andreas Schulze, Michal Inbar-Feigenberg, Neal Sondheimer, Anette Feigenbaum and James Dowling for referring their patients with inherited neurotransmitter disorders for their management. The authors thank Stacy Hewson for genetic counseling for the genetic test results. The authors also thank Duncan Westwood for his work for Institutional Research Ethics Board application of this study.

\section{Statement of Authorship}

DC and GB performed data entry and data analysis, prepared the first draft of the manuscript and approved the final version. RDC and JR carried out diagnosis and follow-up of patients and approval of the final version. SM-A was involved in design and conceptualization of the study, analysis and interpretation of the data and drafting and finalizing the manuscript for intellectual content.

\section{CONFLICTS OF INTEREST}

All of the authors declare no conflicts of interest or competing interests. There were no financial associations.

\section{FUNDING}

This research did not receive any specific grant from funding agencies in the public, commercial or not-for-profit sectors.

\section{Ethical ApProval}

This study is approved by Institutional Research Ethics Board (Approval\#1000057003).

\section{REFERENCES}

1. Pearl PL. Monoamine neurotransmitter deficiencies. Handb Clin Neurol. 2013;113:1819-25.

2. Pearl PL, Wiwattanadittakul N, Roullet JB, Gibson KM. Succinic semialdehyde dehydrogenase deficiency. In: Adam MP, Ardinger HH, Pagon RA, Wallace SE, Bean LJH, Mefford HC, Stephens K, Amemiya A, Ledbetter N editors GeneReviews®. Seattle, WA: University of Washington, Seattle; 1993-2017. Available at: https://www.ncbi.nlm.nih.gov/books/NBK1195/. Accessed October 14, 2017.

3. Opladen T, Hoffmann GF, Blau N. An international survey of patients with tetrahydrobiopterin deficiencies presenting with hyperphenylalaninaemia. J Inherit Metab Dis. 2012;35:963-73.

4. Hyland K. Cerebrospinal fluid analysis in the diagnosis of treatable inherited disorders of neurotransmitter metabolism. Future Neurol. 2006;1:593-603.

5. Mercimek-Mahmutoglu S, Sidky S, Hyland K, et al. Prevalence of inherited neurotransmitter disorders in patients with movement disorders and epilepsy: a retrospective cohort study. Orphanet J Rare Dis. 2015;8:10-2.

6. Akaboshi S, Hogema BM, Novelletto A, et al. Mutational spectrum of the succinate semialdehyde dehydrogenase (ALDH5A1) gene and functional analysis of 27 novel disease-causing mutations in patients with SSADH deficiency. Hum Mutat. 2003;22:442-50.

7. Gibson KM, Christensen E, Jakobs C, et al. The clinical phenotype of succinic semialdehyde dehydrogenase deficiency (4-hydroxybutyric aciduria): case reports of 23 new patients. Pediatrics. 1997;99:567-74.

8. Wassenberg T, Molero-Luis M, Jeltsch K, et al. Consensus guideline for the diagnosis and treatment of aromatic l-amino acid decarboxylase (AADC) deficiency. Orphanet J Rare Dis. 2017;18:12-12.

9. Furukawa Y, Kish S. Tyrosine hydroxylase deficiency. In: Adam MP, Ardinger HH, Pagon RA, Wallace SE, Bean LJH, Stephens $\mathrm{K}$, Amemiya A, editors. GeneReviews ${ }^{\circledR}$. Seattle, WA: University of Washington, Seattle; 1993-2018.

10. Zhang W, Zhou Z, Li X, et al. Dopa-responsive dystonia in Chinese patients: including a novel heterozygous mutation in the GCH1 gene with an intermediate phenotype and one case of prenatal diagnosis. Neurosci Lett. 2017;644:48-54. 